\title{
Black-hole perturbation theory: The asymptotic spectrum of the prolate spin-weighted spheroidal harmonics
}

\author{
Shahar Hod \\ The Ruppin Academic Center, Emeq Hefer 40250, Israel \\ and \\ The Hadassah Institute, Jerusalem 91010, Israel
}

(Dated: May 18, 2021)

\begin{abstract}
Prolate spin-weighted spheroidal harmonics play a key role in black-hole perturbation theory. In particular, the highly damped quasinormal resonances of rotating Kerr black holes are closely related to the asymptotic eigenvalues of these important functions. We here present a novel and compact derivation of the asymptotic eigenvalues of the prolate spin-weighted spheroidal harmonics. Our analysis is based on a simple trick which transforms the corresponding spin-weighted spheroidal angular equation into a Schrödinger-like wave equation which is amenable to a standard WKB analysis. Our analytical results for the prolate asymptotic spectrum agree with previous numerical computations of the eigenvalues which appear in the literature.
\end{abstract}

\section{INTRODUCTION.}

The characteristic dynamics of test fields in black-hole spacetimes has been studied extensively since the pioneering work of Regge and Wheeler [1], see also [2-4] and references therein. An astrophysically realistic model of wave dynamics in black-hole spacetimes should involve a non-spherical background geometry with angular momentum. In terms of the Boyer-Lindquist coordinates, the spacetime of a rotating Kerr black hole is described by the line-element $[5,6]$

$$
d s^{2}=-\left(1-\frac{2 M r}{\rho^{2}}\right) d t^{2}-\frac{4 M a r \sin ^{2} \theta}{\rho^{2}} d t d \phi+\frac{\rho^{2}}{\Delta} d r^{2}+\rho^{2} d \theta^{2}+\left(r^{2}+a^{2}+\frac{2 M a^{2} r \sin ^{2} \theta}{\rho^{2}}\right) \sin ^{2} \theta d \phi^{2},
$$

where $M$ and $a$ are the mass and angular momentum per unit mass of the black hole, respectively. (We use gravitational units in which $G=c=1$ ). Here $\Delta \equiv r^{2}-2 M r+a^{2}$ and $\rho \equiv r^{2}+a^{2} \cos ^{2} \theta$.

In this paper we consider perturbations of the non-spherical Kerr spacetime. The dynamics of a test field $\Psi$ in the rotating Kerr spacetime is governed by the well-known Teukolsky master equation [7]. One may decompose the field as

$$
{ }_{s} \Psi_{l m}(t, r, \theta, \phi)=e^{i m \phi}{ }_{s} S_{l m}(\theta ; a \omega)_{s} \psi_{l m}(r) e^{-i \omega t},
$$

where $\omega$ is the (conserved) frequency of the mode, $l$ is the spheroidal harmonic index, and $m$ is the azimuthal harmonic index. The parameter $s$ is called the spin weight of the field, and is given by $s= \pm 2$ for gravitational perturbations, $s= \pm 1$ for electromagnetic perturbations, $s= \pm \frac{1}{2}$ for massless neutrino perturbations, and $s=0$ for scalar perturbations [7].

With the decomposition (2), $\psi$ and $S$ obey radial and angular equations, both of confluent Heun type [7-11], coupled by a separation constant $A(a \omega)$. The radial Teukolsky equation is given by [7]

$$
\Delta^{-s} \frac{d}{d r}\left(\Delta^{s+1} \frac{d \psi}{d r}\right)+\left[\frac{K^{2}-2 i s(r-M) K}{\Delta}-a^{2} \omega^{2}+2 m a \omega-A+4 i s \omega r\right] \psi=0,
$$

where $K \equiv\left(r^{2}+a^{2}\right) \omega-a m$.

The angular functions $S(\theta ; a \omega)$ are the spin-weighted spheroidal harmonics which are solutions of the angular equation 7 -11]

$$
\frac{1}{\sin \theta} \frac{\partial}{\partial \theta}\left(\sin \theta \frac{\partial S}{\partial \theta}\right)+\left[c^{2} \cos ^{2} \theta-2 c s \cos \theta-\frac{(m+s \cos \theta)^{2}}{\sin ^{2} \theta}+s+A\right] S=0
$$

where $c \equiv a \omega$. The angular functions are required to be regular at the poles $\theta=0$ and $\theta=\pi$. These boundary conditions pick out a discrete set of eigenvalues $\left\{{ }_{s} A_{l m}\right\}$ labeled by the integers $m$ and $l$. [In the $c \rightarrow 0$ limit these angular functions become the familiar spin-weighted spherical harmonics with the corresponding angular eigenvalues $A=l(l+1)-s(s+1)+O(a \omega)$.

The spin-weighted spheroidal harmonics $S(\theta ; c)$ and their corresponding eigenvalues $\left\{{ }_{s} A_{l m}\right\}$ have attracted much attention over the years from both physicists and mathematicians [11 17]. It is worth emphasizing that in order 
to compute the characteristic resonances of black holes [2], one must first compute the (closely related) angular eigenvalues $\left\{{ }_{s} A_{l m}\right\}$ [see Eq. (3)]. In particular, in the framework of semi-classical general relativity, it has been conjectured that the highly damped resonances may shed light on the quantum properties of black holes [18, 19]. For rotating black holes, these highly damped resonances are characterized by $c_{I} \rightarrow \infty$ (where $c_{I} \equiv \Im c$ ).

The asymptotic limit $c_{I} \rightarrow \infty$ of the angular equation (4) was studied in [11, 12] for the $s=0$ case. It was found that the asymptotic scalar eigenvalues are given by

$$
{ }_{0} A_{l m}=[2(l-|m|)+1]\left|c_{I}\right|+O(1) .
$$

As pointed out in [16], the analysis of [11, 12] for the spin-0 case is somewhat incomplete. The analysis of [11, 12] requires the knowledge of the number of zeros of the scalar harmonics in the interval $[0, \pi]$. However, as emphasized in [16], the approximated solution found in [11, 12] for the $c_{I} \rightarrow \infty$ limit is only valid in a region far from the end-points $\theta=0, \pi$. Thus, the analysis of [11, 12] cannot rule out the possible existence of additional zeros of the angular eigenfunctions near the end-points. The possible omission of such zeros would lead to a wrong asymptotic behavior of the prolate (with $c=i c_{I}$ ) eigenvalues, see [16] for details. In this respect the analysis of [11, 12] is not complete. One of the goals of the present paper is to present a more rigorous proof of the formula (5) for the asymptotic scalar eigenvalues.

The asymptotic spectrum of the prolate eigenvalues for the general spin case was first studied in 13]. However, as pointed out in [16, 20], the analysis of [13] for the general spin case is fundamentally flawed (see [16, 20] for details). The numerical results presented in Refs. [16, 20] for the general spin case provide evidence for the asymptotic behavior

$$
{ }_{s} A_{l m}=\gamma\left|c_{I}\right|+O(1)
$$

where the function $\gamma$ depends on the spin-parameter $s$, the azimuthal harmonic index $m$, and the spheroidal harmonic index $l$ [see Eq. (21) below]. The main aim of the present paper is to provide a (simple) analytical proof for the prolate formula (6) in the general spin case.

\section{A COORDINATE TRANSFORMATION}

It proves useful to introduce the coordinate $x$ defined by [17, 21]

$$
x \equiv \ln \left(\tan \left(\frac{\theta}{2}\right)\right),
$$

in terms of which the angular equation (4) becomes a Schrödinger-like wave equation of the form [22]

$$
\frac{d^{2} S}{d x^{2}}-U S=0
$$

where

$$
U(\theta)=(m+s \cos \theta)^{2}-\sin ^{2} \theta\left(c^{2} \cos ^{2} \theta-2 c s \cos \theta+s+A\right) .
$$

Note that the interval $\theta \in[0, \pi]$ maps into $x \in[-\infty, \infty]$. The Schrödinger-type angular equation (8) is now in a form that is amenable to a standard WKB analysis.

\section{THE SPIN-0 (SCALAR) CASE.}

We shall first consider the spin-0 (scalar) case. In this case the effective potential $U(\theta)$ is in the form of a symmetric (invariant under the transformation $\theta \rightarrow \pi-\theta$ ) potential well: in the $c_{I} \rightarrow \infty$ limit it has a local minimum at

$$
{ }_{0} \theta_{\min }=\frac{\pi}{2} \quad \text { with } \quad U\left({ }_{0} \theta_{\min }\right)=-A+O(1)
$$

Regions where $U(\theta)<0$ are characterized by an oscillatory behavior of the wave-function $S$ (the 'classically allowed regions'), while regions with $U(\theta)>0$ (the 'classically forbidden regions') are characterized by an exponential behavior (evanescent waves). The 'classical turning points' are characterized by $U=0$. There is a pair $\left\{{ }_{0} \theta^{-},{ }_{0} \theta^{+}\right\}$of such turning points (with ${ }_{0} \theta^{-}<{ }_{0} \theta_{\min }<{ }_{0} \theta^{+}$), which in the $c_{I} \rightarrow \infty$ limit are located in the near vicinity of ${ }_{0} \theta_{\text {min: }}$

$$
{ }_{0} \theta^{ \pm}=\frac{\pi}{2} \pm \frac{\sqrt{A}}{\left|c_{I}\right|}+O\left(c_{I}^{-3 / 2}\right) .
$$


A standard textbook second-order WKB approximation for the bound-state 'energies' of a Schrödinger-like wave equation of the form (8) yields the well-known quantization condition [23 27]

$$
\int_{x^{-}}^{x^{+}} d x \sqrt{-U(x)}=\left(N+\frac{1}{2}\right) \pi \quad ; \quad N=0,1,2, \ldots,
$$

where $x^{ \pm}$are the turning points [with $U\left(x^{ \pm}\right)=0$ ] of the potential well, and $N$ is a non-negative integer.

Using the relation $d x / d \theta=1 / \sin \theta$, one can write the WKB condition (12) in the form

$$
\int_{0^{-}}^{0^{\theta^{+}}} d \theta \frac{\sqrt{-U(\theta)}}{\sin \theta}=\left(N+\frac{1}{2}\right) \pi \quad ; \quad N=0,1,2, \ldots .
$$

The WKB quantization condition (13) determines the eigenvalues $\{A\}$ of the associated spin-weighted spheroidal harmonics in the large- $c_{I}$ limit. The relation so obtained between the eigenvalues and the parameters $c, m, s$ and $N$ is rather complex and involves elliptic integrals. However, given the fact that in the $c_{I} \rightarrow \infty$ limit the turning points ${ }_{0} \theta^{ \pm}$lie in the vicinity of $\theta=\frac{\pi}{2}$ [see Eq. (11)], one can approximate the integral in (11) by [28]

$$
\int_{0^{-}}^{0^{\theta^{+}}} d \theta \sqrt{-c_{I}^{2}\left(\theta-\frac{\pi}{2}\right)^{2}+A}=\left(N+\frac{1}{2}\right) \pi \quad ; \quad N=0,1,2, \ldots .
$$

Evaluating the integral in (14) is straightforward, and one finds

$$
A(N)=(2 N+1)\left|c_{I}\right|+O(1) \quad ; \quad N=0,1,2, \ldots
$$

for the quantized spectrum. This completes our proof for the prolate asymptotic spectrum in the scalar $(s=0)$ case [29].

\section{THE GENERAL SPIN CASE.}

We shall now consider the general spin case. In this case the effective potential $U(\theta)$ is complex-valued. Its minimum is located at ${ }_{s} \theta_{\min }=\frac{\pi}{2}+\frac{i s}{c_{I}}+O\left(c_{I}^{-2}\right)$, while the two turning points are located at

$$
{ }_{s} \theta^{ \pm}=\frac{\pi}{2} \pm \frac{\sqrt{A}}{\left|c_{I}\right|}+\frac{i s}{c_{I}}+O\left(c_{I}^{-3 / 2}\right) .
$$

A natural generalization of the WKB analysis to the case of complex-valued potentials is provided in [30]: the WKB quantization rule is given by the standard relation

$$
\int_{s \theta^{-}}^{s \theta^{+}} d \theta \frac{\sqrt{-U(\theta)}}{\sin \theta}=\left(N+\frac{1}{2}\right) \pi \quad ; \quad N=0,1,2, \ldots,
$$

which can be approximated near $\theta=\pi / 2$ by [28]

$$
\int_{s}^{s \theta^{-}} d \theta \sqrt{-c_{I}^{2}\left(\theta-\frac{\pi}{2}\right)^{2}-2 i s c_{I}\left(\theta-\frac{\pi}{2}\right)+A}=\left(N+\frac{1}{2}\right) \pi \quad ; \quad N=0,1,2, \ldots .
$$

As emphasized in [30], the integration path between the two complex turning points $\left\{{ }_{s} \theta^{-},{ }_{s} \theta^{+}\right\}$should be chosen such that

$$
\Im\{\sqrt{-U(\theta)}\}=0
$$

along the integration contour [30]. In the $c_{I} \rightarrow \infty$ limit this requirement is easily fulfilled by a straight line (parallel to the real $\theta$-axis) which connects the two turning points (16). Substituting $\theta=\phi-\frac{i s}{c_{I}}$ (where $\phi \in \mathbb{R}$ runs from ${ }_{0} \theta^{-}$to ${ }_{0} \theta^{+}$) into (18) and neglecting terms of order $O(1)$, one finds that along the path (19) the integral (16) can be written as

$$
\int_{0^{-}}^{0^{\theta^{+}}} d \phi \sqrt{-c_{I}^{2}\left(\phi-\frac{\pi}{2}\right)^{2}+A}=\left(N+\frac{1}{2}\right) \pi \quad ; \quad N=0,1,2, \ldots .
$$


This yields

$$
A(N)=(2 N+1)\left|c_{I}\right|+O(1) \quad ; \quad N=0,1,2, \ldots
$$

for the quantized spectrum. This completes our proof for the prolate asymptotic spectrum in the general spin case 29]. It is worth emphasizing that the analytical formula (21) for the prolate asymptotic spectrum agrees with the numerical results presented in [16, 20].

\section{ACKNOWLEDGMENTS}

This research is supported by the Carmel Science Foundation. I thank Uri Keshet, Oded Hod, Yael Oren, Arbel M. Ongo and Ayelet B. Lata for helpful discussions.

[1] T. Regge and J. A. Wheeler, Phys. Rev. 108, 1063 (1957).

[2] H. P. Nollert, Class. Quantum Grav. 16, R159 (1999); E. Berti, V. Cardoso and A. O. Starinets, Class. Quant. Grav. 26, 163001 (2009).

[3] W. H. Press, Astrophys. J. 170, L105 (1971); V. de la Cruz, J. E. Chase and W. Israel, Phys. Rev. Lett. 24, 423 (1970); C.V. Vishveshwara, Nature 227, 936 (1970); M. Davis, R. Ruffini, W. H. Press and R. H. Price, Phys. Rev. Lett. 27, 1466 (1971).

[4] R.H. Price, Phys. Rev. D 5, 2419 (1972); C. Gundlach, R.H. Price, and J. Pullin, Phys. Rev. D 49, 883 (1994); J. Bicák, Gen. Relativ. Gravitation 3, 331 (1972); E. S. C. Ching, P. T. Leung, W. M. Suen, and K. Young, Phys. Rev. Lett. 74, 2414 (1995); E. S. C. Ching, P. T. Leung, W. M. Suen, and K. Young, Phys. Rev. D 52, 2118 (1995); S. Hod and T. Piran, Phys. Rev. D 58, 024017 (1998) arXiv:gr-qc/9712041; S. Hod and T. Piran, Phys. Rev. D 58, 024018 (1998) arXiv:gr-qc/9801001; S. Hod and T. Piran, Phys. Rev. D 58, 044018 (1998) arXiv:gr-qc/9801059; S. Hod and T. Piran, Phys. Rev. D 58, 024019 (1998) arXiv:gr-qc/9801060; S. Hod, Phys. Rev. D 58, 104022 (1998) arXiv:gr-qc/9811032; S. Hod, Phys. Rev. D 61, 024033 (2000) arXiv:gr-qc/9902072]; S. Hod, Phys. Rev. D 61, 064018 (2000) arXiv:gr-qc/9902073; L. Barack, Phys. Rev. D 61, 024026 (2000); S. Hod, Phys. Rev. Lett. 84, 10 (2000) arXiv:gr-qc/9907096; S. Hod, Phys. Rev. D 60, 104053 (1999) arXiv:gr-qc/9907044]; S. Hod, Class. Quant. Grav. 26, 028001 (2009) arXiv:0902.0237]; S. Hod, Class. Quant. Grav. 18, 1311 (2001) arXiv:gr-qc/0008001]; S. Hod, Phys. Rev. D 66, 024001 (2002) arXiv:gr-qc/0201017; R. J. Gleiser, R. H. Price, and J. Pullin, Class. Quant. Grav. 25, 072001 (2008); M. Tiglio, L. E. Kidder, and S. A. Teukolsky, Class. Quant. Grav. 25, 105022 (2008); R. Moderski and M. Rogatko, Phys. Rev. D 77, 124007 (2008); X. He and J. Jing, Nucl. Phys. B 755, 313 (2006); H. Koyama and A. Tomimatsu, Phys. Rev. D 65, 084031 (2002); B. Wang, C. Molina, and E. Abdalla, Phys. Rev. D 63, 084001 (2001); S. Hod, Phys. Rev. Lett. 81, 4293 (1998) arXiv:gr-qc/9812002 ; G. T. Horowitz and V. E. Hubeny, Phys. Rev. D 62, 024027 (2000); E. W. Leaver, Proc. R. Soc. A 402, 285 (1985); S. Hod, Phys. Rev. D 75, 064013 (2007) arXiv:gr-qc/0611004]; S. Hod, Class. and Quant. Grav. 24, 4235 (2007) arXiv:0705.2306]; A. Gruzinov, arXiv:gr-qc/0705.1725; A. Pesci, Class. Quantum Grav. 24, 6219 (2007); S. Hod, Phys. Rev. D 78, 084035 (2008); S. Hod, Phys. Lett. B 666483 (2008) arXiv:0810.5419]; S. Hod, Phys. Rev. D 80, 064004 (2009) arXiv:0909.0314; S. Hod, Phys. Lett. A 374, 2901 (2010) arXiv:1006.4439]; S. Hod, Phys. Rev. D. 84, 044046 (2011) arXiv:1109.4080; S. Hod, Phys. Lett. B 710, 349 (2012) arXiv:1205.5087; B. Mashhoon, Phys. Rev. D 31, 290 (1985); C. J. Goebel, Astrophys. J. 172, L95 (1972); V. Cardoso, A. S. Miranda, E. Berti, H. Witek, and V. T. Zanchin, Phys. Rev. D 79, 064016 (2009); S. R. Dolan and A. C. Ottewill, Classical Quantum Gravity 26, 225003 (2009); S. R. Dolan, Phys. Rev. D 82, 104003 (2010); Y. Décanini, A. Folacci, and B. Raffaelli, Phys. Rev. D 84, 084035 (2011).

[5] S. Chandrasekhar, The Mathematical Theory of Black Holes, (Oxford University Press, New York, 1983).

[6] R. P. Kerr, Phys. Rev. Lett. 11, 237 (1963).

[7] S. A. Teukolsky, Phys. Rev. Lett. 29, 1114 (1972); Astrophys. J. 185, 635 (1973).

[8] A. Ronveaux, Heun's differential equations. (Oxford University Press, Oxford, UK, 1995).

[9] P. P. Fiziev, e-print arXiv:0902.1277; R. S. Borissov and P. P. Fiziev, e-print arXiv:0903.3617; P. P. Fiziev, Phys. Rev. D 80, 124001 (2009); P. P. Fiziev, Class. Quant. Grav. 27, 135001 (2010).

[10] M. Abramowitz and I. A. Stegun, Handbook of Mathematical Functions (Dover Publications, New York, 1970).

[11] C. Flammer, Spheroidal Wave Functions (Stanford University Press, Stanford, 1957).

[12] J. Meixner and F. W. Schäfke, Mathieusche Funktionen und Sphäroidfunktionen mit Anwendungen auf Physikalische und Technische Probleme (Springer-Verlag, Berlin, 1954).

[13] R. A. Breuer, Gravitational Perturbation Theory and Synchrotron Radiation (Lecture Notes in Physics, Vol. 44), (Springer, Berlin 1975).

[14] R. A. Breuer, M. P. Ryan Jr, and S. Waller, Proc. R. Soc. London A358, 71 (1977).

[15] M. Casals and A. C. Ottewill, Phys. Rev. D 71, 064025 (2005).

[16] E. Berti, V. Cardoso, and M. Casals, Phys. Rev. D 73, 024013 (2006); Erratum-ibid. D 73, 109902 (2006).

[17] S. Hod, Phys. Lett. B 717, 462 (2012) arXiv:1304.0529.

[18] S. Hod, Phys. Rev. Lett. 81, 4293 (1998) arXiv:gr-qc/9812002.

[19] U. Keshet and S. Hod, Phys. Rev. D 76, R061501 (2007) arXiv:0705.1179]. 
[20] E. Berti, V. Cardoso and S. Yoshida, Phys. Rev. D 69, 124018 (2004).

[21] H. Yang, D. A. Nichols, F. Zhang, A. Zimmerman, Z. Zhang and Y. Chen, arXiv:1207.4253.

[22] Note that in the quantum-mechanical terminology $-U$ stands for $\frac{2 m}{\hbar^{2}}(E-V)$, where $E, V$, and $m$ are the total energy, potential energy, and mass of the of the particle, respectively.

[23] L. D. Landau and E. M. Liftshitz, Quantum Mechanics, 3rd ed. (Pergamon, New York, 1977), Chap. VII.

[24] J. Heading, An Introduction to Phase Integral Methods (Wiley, New York, 1962).

[25] C. M. Bender and S. A. Orszag, Advanced Mathematical Methods for Scientists and Engineers (McGraw-Hill, New York, 1978), Chap. 10.

[26] B. F. Schutz and C. M. Will, Astrophys. J. 291, L33 (1985); S. Iyer and C. M. Will, Phys. Rev. D 35, 3621 (1987).

[27] Higher order corrections to the asymptotic eigenvalues [see formula (18) below] can be obtained by using a higher-order WKB analysis [26].

[28] Here we have used the relations $\sin \theta=1-\frac{1}{2}\left(\theta-\frac{\pi}{2}\right)^{2}+O\left[\left(\theta-\frac{\pi}{2}\right)^{4}\right]$ and $\cos \theta=-\left(\theta-\frac{\pi}{2}\right)+O\left[\left(\theta-\frac{\pi}{2}\right)^{3}\right]$.

[29] Note that our discrete resonance parameter $N$ corresponds to $l-\max (|m|,|s|)$ of [11, 12].

[30] P. D. Miller, Physica D 152153, 145 (2001). 